\title{
Examining the Potential Role of Minimalist Footwear for the Prevention of Proximal Lower-Extremity Injuries
}

\author{
Michael Ryan ${ }^{1,2}$, Maha Elashi ${ }^{2}$, Richard Newsham-West ${ }^{1}$, and Jack Taunton ${ }^{2}$ \\ ${ }^{1}$ Griffith Health Institute, Griffith University, Southport, Australia \\ ${ }^{2}$ Division of Sport and Exercise Medicine, University of British Columbia, \\ Vancouver, Canada
}

\section{Introduction}

In 2001 Benno Nigg questioned the conventional practice that stated more cushion and more stability equals better running.[1] Nine years after Dr. Nigg's landmark paper, the evidence of change in the running community to greater levels of minimalism is apparent although remains somewhat marginalized. The absence of mainstream acceptance of the minimalist concept could be a result of incomplete evidence in favor of minimalism improving running - through either a combination of injury prevention or performance enhancement. Nevertheless, footwear companies have been motivated to reduce some of the cushion and stability elements in conventional shoes to appreciate some of the theoretical biomechanical advantages barefoot running may offer (such as reduction in external knee moments and rearfoot eversion). [ $[\underline{2}, \underline{3}]$ Despite most major running shoe manufacturers retailing, or conceptualizing, a minimalist shoe of some description little is known about how well this next generation of running footwear achieves one of its basic purported benefits: reducing injury risk.

\section{Purpose of the study}

The purpose of this study was to examine whether the use progressively increasing footwear minimalism can reduce injury risk in runners.

\section{Methods}

Ninety nine runners with neutral or mild pronation were randomly assigned a neutral (NF)(Nike Pegasus 28), partial minimalist shoe (PMF)(Nike Free 3.0 V2) or full minimalist shoe (FMF)(Vibram 5Finger Bikila). Runners underwent baseline testing to record training and injury history, as well as selected anthropometric measurements, before commencing a 12-week training program in preparation for a 10-kilometre event. Outcome measures included number of injury events, Foot and Ankle Disability (FADI) scores and a numerical pain rating scales for foot/ankle, calf/shin, knee, pelvis/groin, low back and overall pain with running.

All data was statistical analysed using IBM SPSS Version 19. Injury incidence data, as well as relative injury risk across footwear conditions were calculated. A Kaplan-Meier survival analysis was carried out using each injury as an event. A 2-way analysis of variance (ANOVA) to determining whether there are significant main effects for time and footwear condition, as well as an interaction effect, for all three dependent variables. The following variables were included in the model as covariates: age, body mass index, severity of prior history of running injury, Hallux ROM, ankle ROM, Q-angle, and prior proportion running on road. 


\section{Results}

Overall there were 23 injury events recorded over the 12 week period resulting in an injury rate of $23.2 \%$. There were more injuries in the PMF group than the NF group contributing to a $192 \%$ relative risk of injury in the PMF group compared to neutral. Comparatively, there was a $54 \%$ relative risk of injury in those runners in the FMF condition relative to the neutral shoe. Kaplan-Meier analysis reported a significant difference in the number of injury events over time across footwear condition. Based on injury event data, there is a higher likelihood of experiencing an injury with minimalist footwear, particularly with the partial minimalist condition.
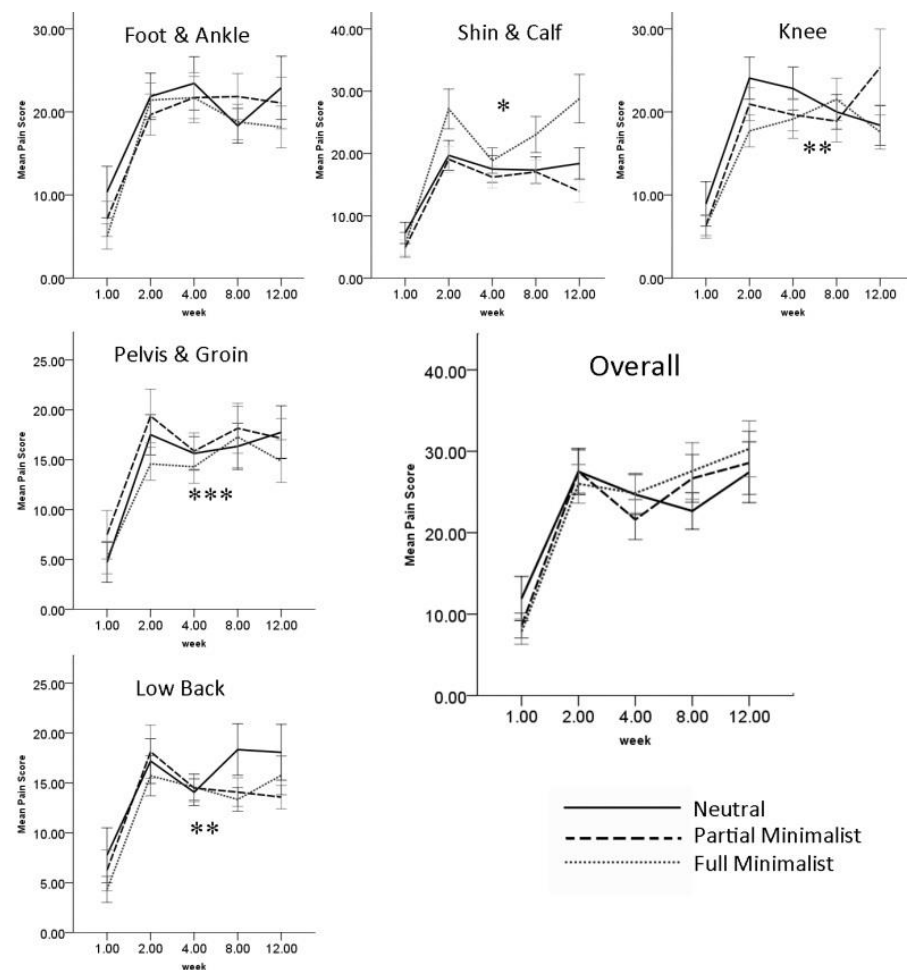$$
\text { week }
$$

Figure 1 - Ensemble graph for numerical pain rating scale outcomes across anatomical region and overall pain rating. $*, * *, * * *$ represent a significant difference between the full minimalist footwear (FMF) group and both neutral footwear (NF) and partial minimalist footwear (PMF) groups, the FMF and NF group, and the PMF and FMF group, respectively at a $\mathrm{p}<0.05$ level.
Outcomes from the numerical pain scale also report anatomical region differences in pain perception across footwear conditions over the 12-week training period (Figure 1). In particular, runners in the FMF group reported fewer knee and low back pain than the NF group, and less pelvis and groin pain than PMF group. However, the FMF group did report greater calf and shin pain.

\section{Discussion and conclusion}

Running in minimalist footwear appears to increase the likelihood of experiencing an injury and running related pain in runners otherwise new to this footwear category while training for a $10 \mathrm{~km}$ event. Using a full minimalist design; however, may reduce pain at the knee, hip and pelvis, and lower back suggesting there be merit in using a full minimalist design for injury prevention with appropriate preconditioning of soft-tissue in the shin and calf.

1. Nigg, B.M., The role of impact forces and foot pronation: a new paradigm. Clin J Sport Med, 2001. 11(1): p. 2-9.

2. DeWit, B., D. DeClercq, and P. Aerts, Biomechanical analysis of the stance phase during barefoot and shod running. J Biomech, 2000. 33(3): p. 269-78.

3. Squadrone, R. and C. Gallozzi, Biomechanical and physiological comparison of barefoot and two shod conditions in experienced barefoot runners. J Sports Med Phys Fitness, 2009. 49(1): p. 6-13. 